\title{
Particular aspects regarding the effects of whole body vibration exposure
}

\author{
Mihaela Picu, ${ }^{1, *}$, Laurentiu Picu ${ }^{1}$ \\ 1 "Dunarea de Jos" Galati University, Romania
}

\begin{abstract}
This paper analyses the influence of whole-body vibrations on human performance; for this it was investigated how a group of men (20-29 years of age) and a group of woman (21-31 years of age) answered to specific requirements after being subjected to vertical vibrations under controlled laboratory conditions for 10-25 min. The vibrations were generated by a vibrant system with known amplitudes and frequencies. Accelerations were measured with NetdB - complex system for measuring and analysing human vibration and they were found in the range $0.4-3.1 \mathrm{~m} / \mathrm{s}^{2}$. The subjects' performances were determined for each vibration level using specific tests. It can be concluded that exposure to vibrations higher than those recommended by ISO 2631 significantly disrupts how subjects responded to tests requirements.
\end{abstract}

\section{Introduction}

Our body is exposed to vibrations in the occupational environment because of several mechanisms such as construction equipment, heavy equipment (grinders, pick-hammers) and electric hand tools. It has been shown that vibrations lead to the disruption of the muscular and bone systems of both arms, of the neck and of the back $[1,2]$.

Occupational WBV exposure, especially when chronic, is suspected to cause adverse health effects such as fatigue, lower back pain, vision problems, interference with or irritation to the lungs, abdomen, or bladder, and adverse effects to the digestive, genital/urinary, and female reproductive systems [3]. In addition to the problems of muscular and bone systems, the exposure of the whole body to occupational vibrations also creates psychomotor, physiological and psychological problems [4].

Contact with a vibrating machine transfers the vibration energy to the human body. Depending on the type of exposure, vibrations can affect much of the worker's body or only a certain organ. The effect of vibration exposure also depends on its frequency. Each body organ has its own resonance frequency. If the exposure occurs at that resonance frequency or near one, the resulting effect is increased [5].

The negative effect of vibrations transmitted by moving equipment on operators have been studied by

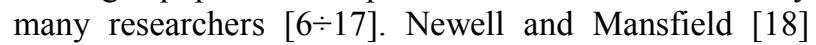
found out on a group of 21 subjects exposed to vibrations of $1 \div 20 \mathrm{~Hz}$ and placed in various positions, that their work performances dropped significantly, the reaction times increased and the workload decreased. Including the armrest improved the working conditions, thus the performance.
The frequency dependence of discomfort caused by vertical mechanical shocks has been investigated with 20 seated males exposed to upward and downward shocks at 13 fundamental frequencies and 18 magnitudes. The rate of growth of discomfort with increasing shock magnitude depended on the fundamental frequency of the shocks, so the frequency dependence of equivalent comfort contours varied with shock magnitude. The rate of growth of discomfort was similar for acceleration and force, upward and downward shocks, and lower and higher magnitude shocks [19].

Liu, Qiu and Griffin [20] analyzed fourteen male subjects who stayed on a rigid seat with feet hanging while exposed to random vertical vibration $(0.5$ to 20 $\mathrm{Hz})$ at three vibration magnitudes $\left(0.25,0.5\right.$, and $1.0 \mathrm{~ms}^{-2}$ r.m.s.). The frequency of the principal resonance in the vertical apparent mass was higher at the front thighs $(8$ to $10 \mathrm{~Hz}$ ) than at the ischial tuberosities (around $5 \mathrm{~Hz}$ ). There was greater nonlinearity in the vertical apparent mass measured at the front thighs than at the ischial tuberosities.

Apparent mass frequency response functions of the seated human body have been measured with random vibration in the vertical direction at frequencies up to 20 $\mathrm{Hz}$.

Relative movement between the feet and the seat was found to affect the apparent mass at frequencies below resonance, particularly near zero-frequency.

The magnitude of the vibration had a consistent effect: the resonance frequency decreased from about 6 to $4 \mathrm{~Hz}$ when the magnitude of the vibration was increased from 0.25 to $2.0 \mathrm{~ms}^{-2}$ r.m.s. The apparent masses of all the subjects were remarkably similar when normalized with respect to sitting weight [21].

Matsumoto and Griffin [22] analyzed the influence of the posture of the legs and the vibration magnitude on

\footnotetext{
* Corresponding author: mihaelapicu@yahoo.com
} 
the dynamic response of the standing human body exposed to vertical whole-body vibration has been investigated. Motions were measured on the body surface at the first and eighth thoracic and fourth lumbar vertebrae (T1, T8 and L4), at the right and left iliac crests and at the knee. Twelve subjects took part in the experiment with three leg postures (normal, legs bent and one leg), and five magnitudes of random vibration $\left(0.125-2.0 \mathrm{~ms}^{-2}\right.$ r.m.s.) in the frequency range till $30 \mathrm{~Hz}$. The main resonance frequencies of the apparent masses at $1.0 \mathrm{~ms}^{-2}$ r.m.s. differed between postures: $5.5 \mathrm{~Hz}$ in the normal posture, $2.75 \mathrm{~Hz}$ in the legs bent posture and 3.75 $\mathrm{Hz}$ in the one leg posture.

Multi-directional vibration was also investigated. In the biodynamic research, subjects were exposed to 0.55 , 1.75 and $2.25 \mathrm{~ms}^{-2}$ r.m.s. vibration levels in the $0.5-40$ $\mathrm{Hz}$ frequency domain. The influence of sitting position on human body behaviour under two axial vibrations was also examined. Data analysis showed that the human body behaviour under two-directional random vibrations could not be approximated by superposition of onedirectional random vibrations [23].

\section{Materials and methods}

Subjects: The experiment involved 6 women and 6 men (to investigate variability between genders) aged between 20-31 years, in good health. Each participant was asked to complete a health screening questionnaire, to give written consent for tests and was instructed in writing concerning the experiment. Participants were informed that they may abandon the experiment at any time for any reason.

Experimental conditions: Experiments were made inside the laboratory, the temperature and humidity have been set to $22^{\circ} \mathrm{C}$, respectively $60 \%$.

The vibrations were transmitted by Modal Exciter Brüel \& Kjær type 4827 and were analysed with NetdB Complex system to measure human vibration using PCB Piezotronics 356A16 - Triaxial Accelerometers. Each experiment lasts 10-25 minutes.

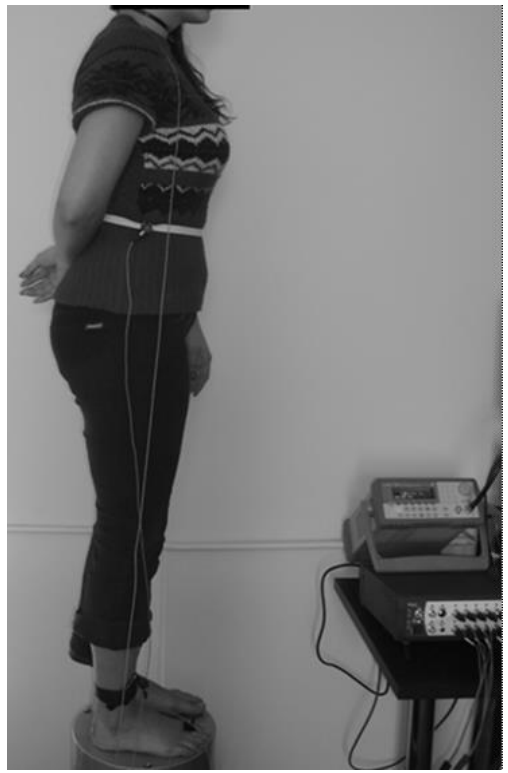

Fig. 1. The subject stands on the vibrant system
The values of vibrations level transmitted to the subject were chosen as close as possible to those in real conditions: frequency $v=2.65 ; 4.55 ; 8.25 \mathrm{~Hz}$; acceleration $a_{\text {r.m.s }}=0.425 ; 2.755 ; 3.100 \mathrm{~m} / \mathrm{s}^{2}$; vibration time $\mathrm{t}=10 ; 20 ; 25 \mathrm{~min}$.

There was also a control case: $\mathrm{a}_{\mathrm{r} . \mathrm{m} . \mathrm{s}}=0$ (a subject placed on the vibrant system - but it does not work without the subject being informed of this situation).

Two cases were studied: subject standing on the vibrant system and subject placed on the vibrating system in sitting position (Fig. 1 and 2).

Discomfort determination using Likert scale: The first part of the experiment was to determine the subjects' discomfort exposed to stressors, using the Likert scale: Very strongly: $4 \div 5$; Strongly: $3 \div 4$; Slightly: $2 \div 3$; A little: $1 \div 2$; Not at all: $0 \div 1$. Each subject was given a score for what he felt after the experiment ended [24].

Tests of human performance: Subjects answered 3 types of tests: Logical and mausing (locate a point with the mouse pointer) tests, Psychological Test, Stress tests.

\section{Results and discussions}

In order to determine how to quantify the action of these external factors on human performance, it will be considered that each of the stressors equally concurs to subject's disturbance: $S=1 / 3 \cdot a_{\text {r.m.s. }}+1 / 3 \cdot v+1 / 3 \cdot t$ [A.U.] , where $\mathrm{S}$ is the stress corresponding to the considered case [24].

The performance $(\mathrm{P})$ of a subject was determined as follows: $\mathrm{P}=0.5 \cdot[0.5 \cdot \mathrm{CP}+\mathrm{LM}]$ [A.U.], where $\mathrm{CP}$ is cognitive performance measurement: $\mathrm{CP}=$ $0.5 \cdot($ Psy $+\mathrm{ST})$; LM is Logical and mausing tests; Psy is Psychological Test and ST is Stress tests [24].

In order to analyze how subjects responded to the three stressors, different combinations were made between them. In all experiments sets were randomly introduced "placebo" situations (where $v=0$ and $\mathrm{a}_{\text {r.m.s. }}=0$ and $\mathrm{S}=3.333,6.666$ and 8.333 respectively).

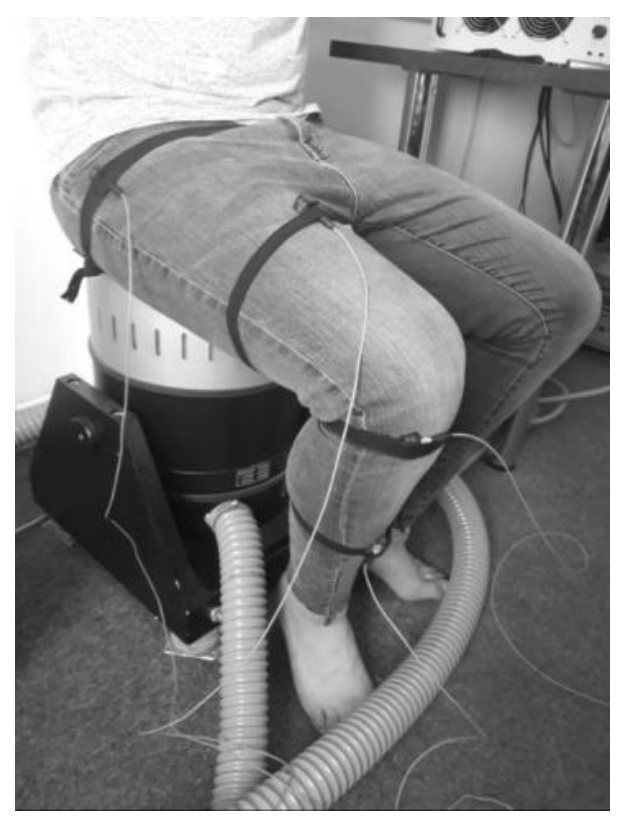

Fig. 2. The subject is sitting on the vibrant system 
The following symbols are used in all graphical

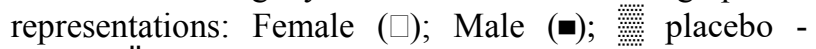
female; \|placebo - male.

Set 1 Figures 3-5 show the effect of acceleration r.m.s. and vibration time on human performance (for constant frequencies).

Figure 3 shows that as the acceleration and vibration time increases, the stress value increases. Women proved to be more resistant than men for lower stressors (performance values are higher). In cases 1,5 and 8 the "placebo" cases appear. Even if in these situations the subjects are not subject to vibration, the test results were not very good (with higher values performance for males).

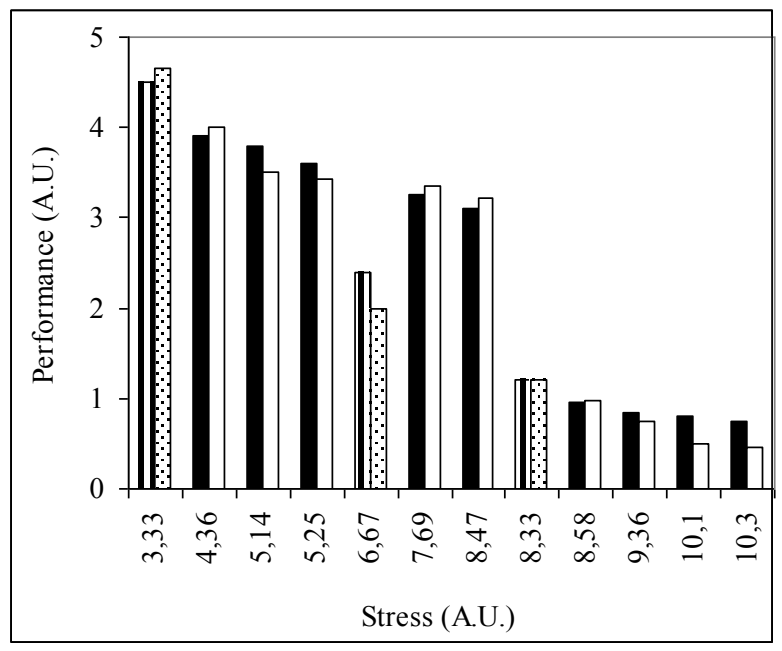

Fig. 3. Effect of acceleration r.m.s. and time on human performance $(v=2.65 \mathrm{~Hz})($ Cases $1 \div 9)$



Fig. 4. Effect of acceleration r.m.s. and time on human performance $(v=4.55 \mathrm{~Hz})($ Cases $10 \div 18)$

Figure 4 shows the "placebo" cases in situations 1, 5 and 7. Performance values for case $7(\mathrm{~S}=8.33333)$ are lower than those similar in Figure 3. Women also had better results than men for lower stressors.

Both for women and men there is a sudden decrease in performance at high stressors.

Figure 5 shows that subjects have high performance values in almost all situations. The test values for "placebo" situations are unexpectedly small, especially for situations 3 and 6 . It may be concluded that fatigue is beginning to develop.

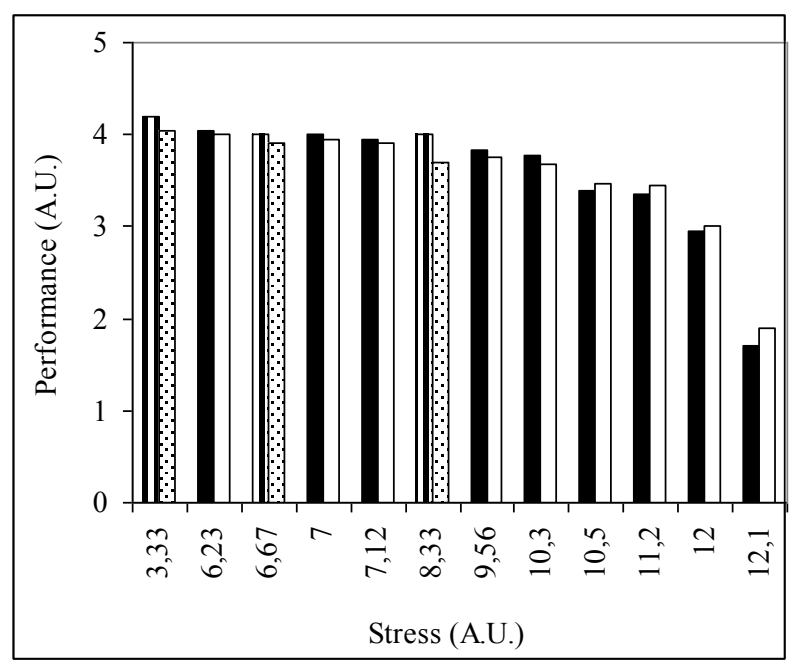

Fig. 5. Effect of acceleration r.m.s. and time on human performance $(v=8.25 \mathrm{~Hz})($ Cases $19 \div 27)$

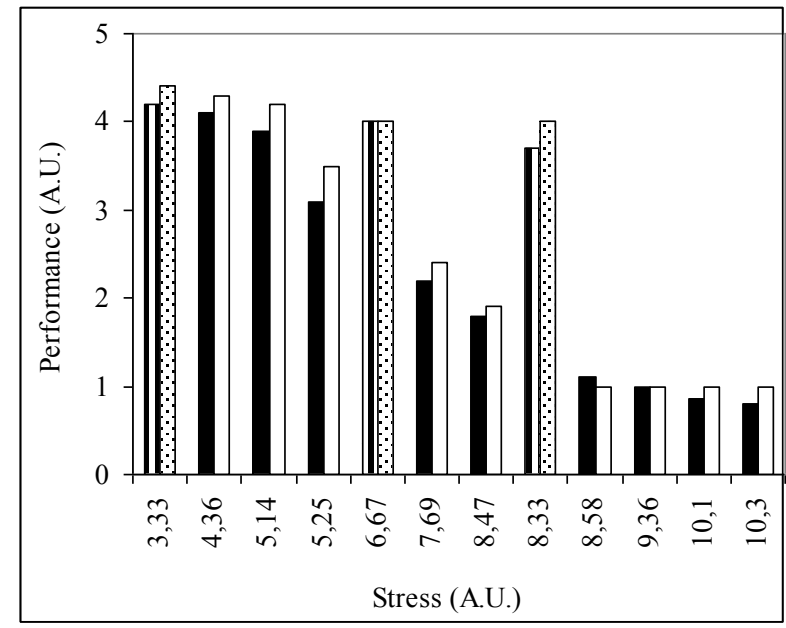

Fig. 6. Effect of frequency and time on human performance $\left(a_{\text {r.m.s. }}=0.425 \mathrm{~m} / \mathrm{s}^{2}\right)($ Cases $28 \div 36)$

Set 2 In sets 2 and 3 we found the following: each combination represents - from a mathematical point of view - the same situation as one of the previous one. However, the reaction of the subjects is different, namely, the higher the stress values, the subjects considered that they "feel" the "placebo" more intense.

Figures 6-8 show the effect of frequency and vibration time on human performance (for constant acceleration r.m.s).

Figure 6 shows that - with the increase of stressors values - women achieved better results in tests than men, even in "placebo" situations (1,5 and 8).

Figure 7 shows a slightly different situation from the precedents: for low stress levels, test results are good and considerably equal both for women and men.

Also for "placebo" situations, the same thing is found. From the middle of the experiment to the end it is seen that men get 2 values of unexpectedly good performances; afterwards these values drop sharply. Instead, women maintain some constancy. 
Figure 8 shows that the first dropouts occurred: women - in the last 3 experiments and men in the last experiment.

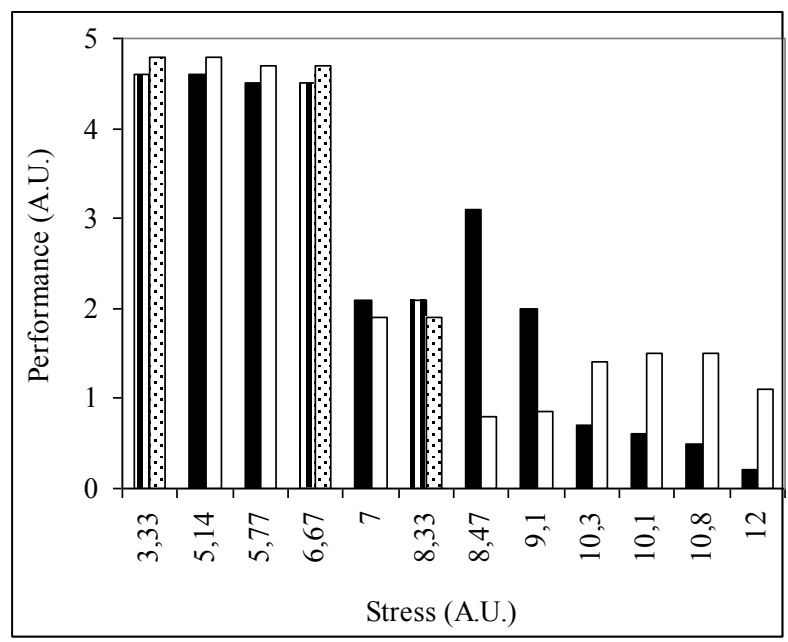

Fig. 7. Effect of frequency and time on human performance $\left(a_{\text {r.m.s. }}=2.755 \mathrm{~m} / \mathrm{s}^{2}\right)($ Cases $37 \div 45)$

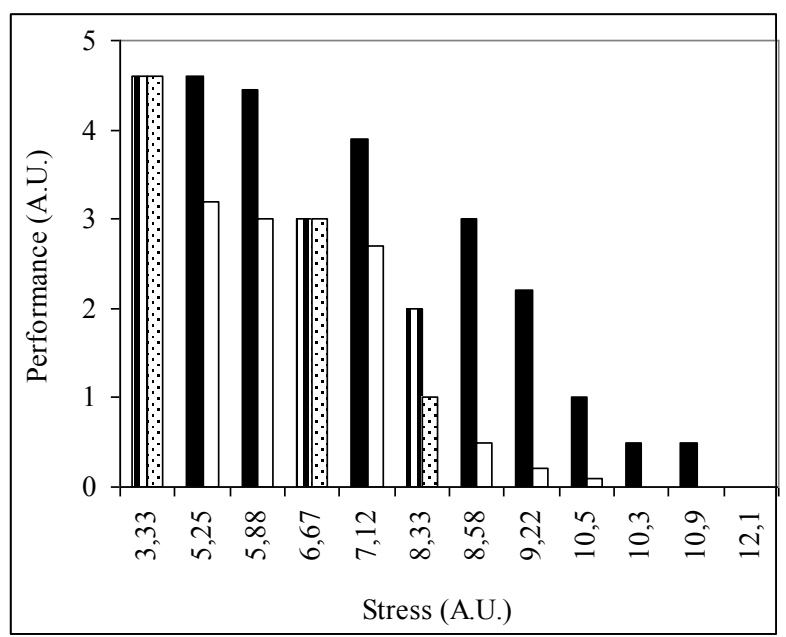

Fig. 8. Effect of frequency and time on human performance $\left(a_{\text {r.m.s. }}=3.100 \mathrm{~m} / \mathrm{s}^{2}\right)($ Cases $46 \div 54)$

Even the results for the third "placebo" are extremely modest. The men had much better results than women.

It is clear that women's fatigue has occurred and, implicitly, the power of concentration needed to solve the tests.

Set 3 Figure 9 shows the effect of frequency and acceleration r.m.s. on human performance. Figure 9 shows that in the first case men had a higher test score; in the following cases, women had a higher score, but in the latter case, women gave up.

There is no logical correlation between the level of stress and the performance of subjects. It can be seen that at a higher stress (9.1) better performance has been achieved than at lower stress value (7.69). In the latter case, women gave up.

In these last experiments, women gave up on the first attempt and the men gave up the last experiment. There have been no "placebo" situations here. The test results were modest and had equal values in both situations.
The effects of frequency and acceleration on human performance ( $\mathrm{t}=20 \mathrm{~min}$ and $25 \mathrm{~min}$ ) (Cases $64 \div 72$ and Cases $73 \div 81$, ) were no longer represented due to the fact that there were many abandonments.

\section{Conclusions}

After analysing the results obtained in the tests it is ascertained that, as the intensity of the stressors increases, the performance decreases. There is, however, a situation (cases 10-18) where performance has increased. It could be an explanation that subjects already had the experience of the previous 9 cases, becoming more careful in solving the tests.

For Cases 1-36 (Figures 3-6), performance (P) can be considered as parabolic dependence of stress $(\mathrm{S}): \mathrm{P}=$ $\mathrm{a} \cdot \mathrm{S}^{2}+\mathrm{b} \cdot \mathrm{S}+\mathrm{c}\left(\mathrm{R}^{2} \approx 0,8\right)$, where $\mathrm{a}, \mathrm{b}$ and $\mathrm{c}$ are constants that depend on each individual situation.

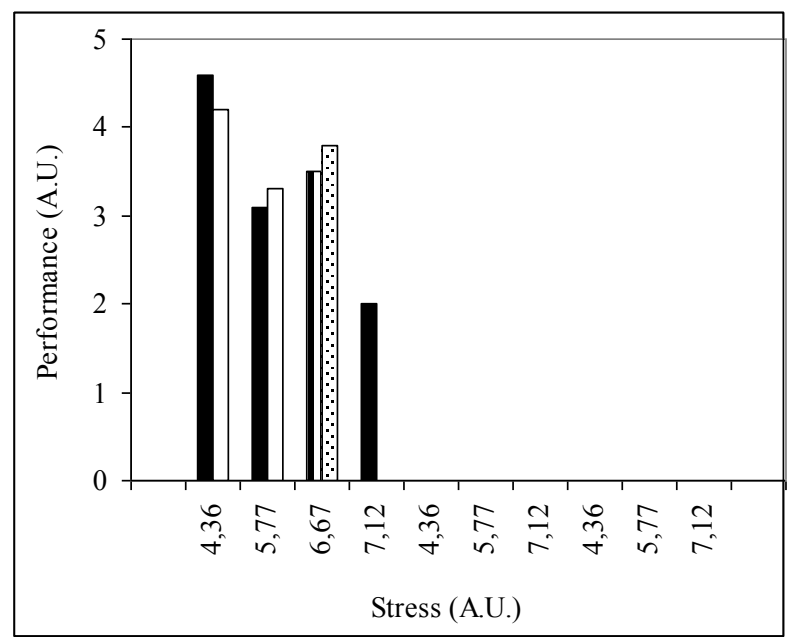

Fig. 9. Effect of frequency and acceleration on human performance $(t=10 \mathrm{~min})($ Cases $55 \div 63)$

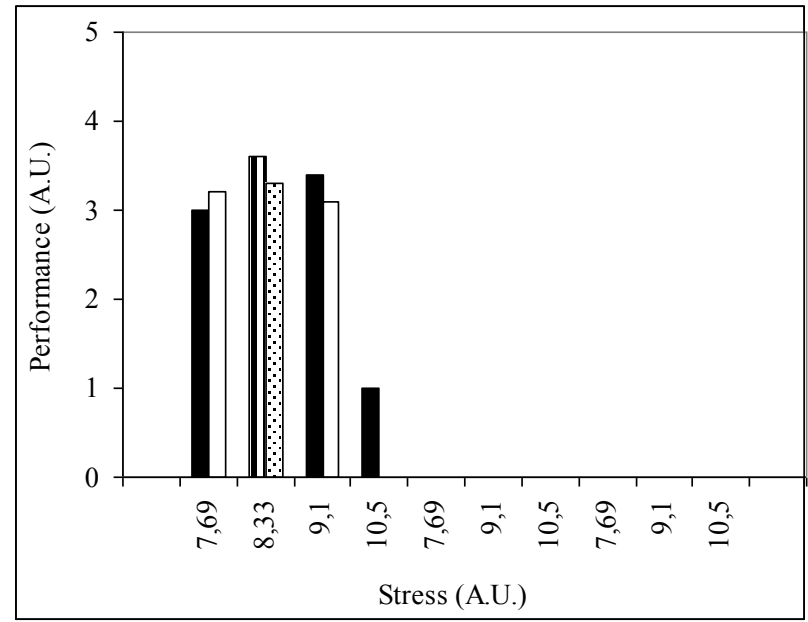

Fig. 10. Effect of frequency and acceleration on human performance $(\mathrm{t}=20 \mathrm{~min})($ Cases $64 \div 72)$

For cases 37 - 45 (Figure 7), there was no dependence between performance $(\mathrm{P})$ and stress $(\mathrm{S})$. For cases 46-54 (Figure 8 ), there was also a parabolic dependence, with the observation that $(\mathrm{R} 2 \approx 0.93)$. Starting Case 55 to the 
end (Case 81), the performances of the subjects were becoming more modest, the results obtained being not proportional to the induced stress (Figures $9-11$ ). It should be emphasized that all these cases represent a repetition of previous cases, so the lack of concentration of the subjects can be attributed to physical and psychological fatigue.

In most situations, women and men have obtained similar results in tests. In the latter cases, part of the subjects abandoned the experiments: for Cases 46-54 (Figure 8) and 73-81 (Figure 11), women abandoned 3 experiments and men 1 experiment; for Cases 55-72, women abandoned 1 experiment.

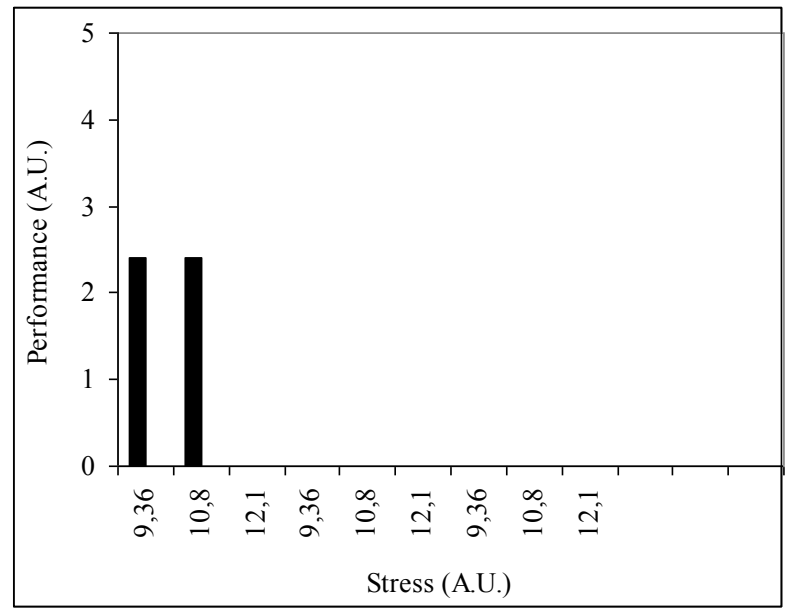

Fig. 11. Effect of frequency and acceleration on human performance $(t=25 \mathrm{~min})($ Cases $73 \div 81)$

We must emphasize that the subjects who abandoned were those who were younger.

Smokers are among those who have abandoned, but there were also non-smokers who have given up.

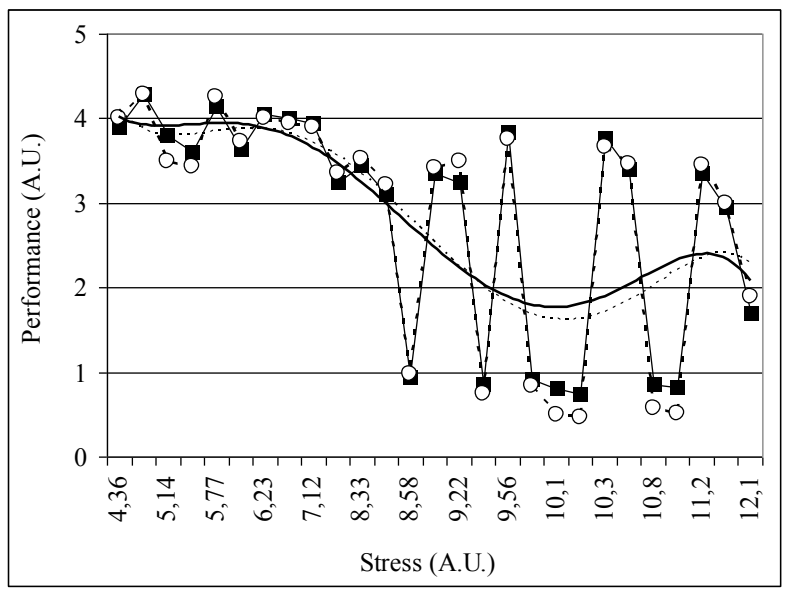

Fig. 12. Human performance variation depending on vibration parameters, for all sets of experiments $(-\overline{-})=$ male; $\left(--_{-}-\right.$ - ) = female; (-) fitting for men; (- $)$ fitting for female

In conclusion, there were no significant differences between men and women (Figure 12). What really influences people's performance is personality, as well as personal problems, fatigue. It is very important to know the problems of each worker in order to know what professional tasks he / she can do.

\section{References}

1. C.H. Lewis, M.J. Griffin, J. Sound Vib. 215: 915926 (2005)

2. M. Cardinale, M.H. Pope, Acta Physiol Hung. 90(3):195-206 (2003)

3. H.W. Paschold, A.G. Mayton, Professional Safety 56: 30-35 (2011)

4. N.J. Mansfield, M.J. Griffin, J. Sound Vib. 215, 813-825 (2005)

5. R. Lundström, P. Holmlund, J. Sound Vib. 215: 801-812 (2007)

6. M. Bovenzi, F. Ronchese, M. Maurom, Int. Arch. Occup. Environ. Health 84, 325-334 (2011)

7. H. Dupuis, G. Zerlett, The Effects of Whole-Body Vibration, Ed. Springer-Verlag Berlin Heidelberg (2012)

8. L. Gerhardsson, L. Burstrom, R. Lundstrom, T. Nilsson, J. Occup. Med. Toxicol. 8(1):8 (2013)

9. G.S. Paddan, N.J. Mansfield, C.I. Arrowsmith, A.N. Rimell, S.K. King, S.R. Holmes, Ergonomics 55(8): 923-936 (2012)

10. J.J. Pilcher, E. Nadler, C. Busch, Ergonomics 45(10): 682-698 (2002)

11. M. Picu, J. Mater. Sci. Eng. A, 4(9): 315-320 (2014)

12. M. Picu, Adv. Mater. Res. 837: $452-457$ (2014)

13. M. Picu, $24^{\text {th }}$ International Conference of Noise and Vibration, Nis, Serbia: 209-216 (2014)

14. M. Picu, Appl. Mech. Mater. 325-326: 152-157 (2013)

15. M. Picu, Int. J. Mod. Manuf. Tech. V(1): 87-90 (2013)

16. M. Picu, Int. J. Mod. Manuf. Tech. IV(2): 61-66 (2012)

17. J. Village, C. Trask, Y. Chow, J.B. Morrison, M. Koehoorn, K. Teschke, Ergonomics 55(4): 415-424 (2012)

18. G.S. Newell, N.J. Mansfield, Int. J. Ind. Erg. 38(56): 499- 508 (2008)

19. Z. Zhou, M.J. Griffin, Ergonomics 60(3) (2017)

20. C. Liu, Y. Qiu, M.J. Griffin, $50^{\text {th }} U K$ Conference on Human Responses to Vibration, University of Southampton, England (2015)

21. T.E. Fairley, M.J. Griffin, J. Biomech. 22(2): 81-94 (1989)

22. Y. Matsumoto, M.J. Griffin, J. Sound Vib. 212(1): 85-107 (1998)

23. M. Demić, J. Lukić, Ž. Milić, J. Sound Vib. 253(1): 109-128 (2002)

24. M. Picu, JMEST 2(9) (2015) 